\title{
The Relationship Between Mindfulness And Mental Health: A Mediating Model of Sense of Purpose In Life Among Graduate Students
}

\author{
Yibin Wang \\ Jiangsu Second Normal University \\ Tian Tian (D466199671@qq.com) \\ Shaanxi University of Technology \\ Junjie Wang \\ Jiangsu Second Normal University
}

\section{Research Article}

Keywords: mindfulness, sense of purpose in life, mental health, graduate student

Posted Date: June 21st, 2021

DOI: https://doi.org/10.21203/rs.3.rs-590135/v1

License: (c) (i) This work is licensed under a Creative Commons Attribution 4.0 International License.

Read Full License 


\section{Abstract}

The purpose of the present study is to examine the relationship between mindfulness and mental health of graduate students and the mediating effects of sense of purpose in life on mindfulness and mental health.The participants include 419 graduate students from 6 universities in China. The Hayes Process is adopted to analyze the effects of the sense of purpose in life on mindfulness and mental health of graduate students.The results reveals that mindfulness can predict the mental health of graduate students positively and significantly. The sense of purpose in life is found to mediate the relationship between mindfulness and mental health.In further moderated mediation analyses,the effect of mindfulness on mental health can be adjusted by family economic condition. The type of degree can adjust the effect of mindfulness on sense of purpose,and professional interest can adjust the mediating effect of sense of purpose.Finally,this study discusses several empirical and methodological implications of the findings.

\section{Introduction}

At present, mental health, as an important part of mental health,is widely recognized and highly valued by all sectors of society.To an individual,not only is it the aggregation of cognition,emotion,volition and behavior in the social environment,but also it includes the judgment of the meaning and value of the individual's life state. Mental health is a harmonious state without psychological problems, which enables individuals to effectively explore available positive forces,helping to pursue higher self-growth.Many theoretical and practical achievements have been made in previous studies of mental health of different groups. However,in the field of higher education,the mental health of graduate students becomes severer and severer,because of the combined impact of the continuous expansion of the graduate students' enrollment and multiple pressures.

\section{Mental health of graduate students}

The graduate students are a core group for the country's development.Not only do universities continue to optimize their training model,but also they need to pay attention to students' mental health and other nonintellectual factors.Previous studies still have many obvious deficiencies in the field of mental health.On the one hand, according to 'The Dual-Factor Model of Mental Health', the mental health of an individual is mainly characterized by positive factor indicators and negative factor indicators (Suldo,Shaffer\&Doll,2008).The previous studies often used Self-reporting Inventory (SCL-90) to measure the mental health of graduate students. Until now,these survey results have only covered the negative part of the psychology of graduate students, such as anxiety and depression and lacked the attention to positive factors(Zhou Chao et al., 2019, Dong Hao eta., 2020). And positive psychology,as an important direction of current psychological research,can not be ignored in promoting the all-round development and healthy growth of individuals. On the other hand, the existing researches are "What factors can affect mental health of graduate students?"The compensating model is usually used to explain people's mental health state,representing the compensatory activity of positive characteristics 
toward negative factors.According to the empirical research, a variety of positive traits can be used to inhibit the generation of negative factors.For instance,perceived social support can inhibit the production of the negative psychological characteristics of graduate students (Zhang Wenlong, 2019).The"problembased"research concept causes circumscribed and rapid educational practices, which was demonstrated in previous studies. The mental health education for graduate students should explore practical methods for human development,which is a psychological development model representing the activity of causing positive characteristics to influence positive factors in order to achieve the self-operation of the psychological development of graduate students. Therefore,the present study will choose positive postgraduate's mental health model as the research variable to analyze.The model contains three dimensions: positive emotional experience, good psychological quality and adequate social support(O.W,G.A,2020;Liu W.L,2017).To sum up,this study takes graduate students as the research object to explore the positive psychological factors that affect the mental health of graduate students and their interaction mechanism, which has unique theoretical and practical significance.

\section{Relationship between Mindfulness and Mental Health of graduate students}

Mindfulness was proposed in the 1970s by psychologist Ellen Lange and others,which is an individual's cognitive way to keep open to new things.It includes three dimensions: seeking new ideas,creating new ideas and focusing.Compared with mindlessness, it is a positive and hardworking conscious thinking mode and a flexible mental state.So far, research on mindfulness has been widely used in the clinic field,personality field,industry and organization field, and positive psychology field.In recent years,scholars have also tried to apply mindfulness to the education field.Studies have shown that graduate students had higher self-expectation and motivation for achievement,and they face the pressures of academic background, academic development,employment pressure, and interpersonal difficulties, at the same time.Such multiple pressures lead to producing widespread negative emotions, and the number of postgraduates with mental health problems is dramatically increasing year after year.The Research has shown that mindfulness training will help people to positively promote individual mental health and working (learning) performance. People with a higher level of mindfulness will have a more clear understanding of themselves and the current environment,and will be easier to clearly discover the essence and solution of the problem in a more rational way.In summary,mindfulness can promote better development of graduate students' mental health. The present study aims to improve the mental health of graduate students by promoting graduate students' level of mindfulness,which enable them to adopt a non-judgmental and accepting attitude towards internal and external stimuli,re-evaluate specific events by a positive mode, reduce negative emotions and give the events new meaning, thus generating positive emotions to relieve stress.

\section{The mediating role of the sense of purpose in life}

The purpose of life is a general intention that has individual subjective will.It is meaningful to ego and can affect the external environment(Damon eta, 2003). The purpose of life is usually understood as a high-dimensional psychological structure integrated and constructed to satisfy needs and desires and 
pursue happiness. Its core is interpreted as the behavioral intention of seeking pleasure and avoiding suffering in order to obtain happiness by shunning misfortune.The purpose in life is a psychological state leading to positively develop,after ignoring individual advantages,social capital and other different influencing factors. The purpose of life can help us understand the origin and reality of human health,the success and happiness during career developmental progression.(Bronk,2014, Hill,Burrow \& Sumner,2013; Kashdan \& McKnight,2009).It can clearly be seen that both theoretical and empirical research have gradually clarified the key role of life purpose in the active development of people (Langongrui,2017)."The Sense of purpose of life" measures whether an individual's purpose in life is stable and sustainable and roots from the active exploration of internal and external ego in environmental events and social learning.As a cognitive mode, mindfulness plays an important role in the exploration of purpose in life .It's the main reason why this study takes the sense of purpose in life as an intermediary variable. According to the Self-decide Theory (Dec i Edward L, 1975),the internal dynamic model was appeared to explain self-behavior,which is tendentiously selected after self-cognition and environmental information have been fully integrated.Mindfulness is a behavioral processes where new information is accepted through multiple perspectives and categories are reconstructed by multi-perspective approaches, which is an indispensable characteristic in the process of self-decision.Individual behavior is driven through a broader goal called "The purpose in life".It is the development intention to point and transcend ego,having the same positive impact on the construction of postgraduates' mental health.On the one hand, mindfulness promotes the efficient operation of the internal drive to explore the value of life by ascent of cognition.When the purpose in life is moulded, the efficiency of self-exploration is increased to seek information from environment.At the same time,the threshold of awareness is opened up to increase the sensitivity that can clarify life purpose.On the other hand,a clear purpose in life has a positive effect on the positive factors of students' mental health.It can help get more positive emotional experience and cultivate positive psychology and accumulate social support when graduate students with a distinct sense of purpose in life have positively predicted life events in order to experience abundant positive emotions(Özkan Çıkrıkçı, 2020). Meanwhile, positive psychological qualities for individuals can be cultivated by the sense of purpose in life,such as subjective well-being, psychological adaptation,selfregulation,etc,which will promote the cultivation of individuals when the pursuit of self-improvement is improved.In addition, adolescents' peer interaction and parenting model are directly affected by the purpose in life,obtaining different levels of social support ( $\mathbb{Q} \mathbb{Q}, \mathbb{Q}, \mathbb{Q}, 2020)$. The purpose of life has a positive effect on the perceived social support had confirmed(Mengxuan Zhang et al, 2019)ם

In summary, this study constructs an intermediary model based on The Positive-Factor Model of Mental Health and the Self-decide Theory to investigate whether it is possible intermediary mechanism.It is possible that the sense of purpose in life may affect between the mindfulness and mental health of graduate students.

\section{Method}

\section{Participants}


The study adopts a convenient random sampling method,and collects a total of 419 graduate students' questionnaires from 6 universities including Shaanxi University of Technology,Shaanxi University of Science and Technology,Lanzhou University of Technology,Xiangtan University, Nanjing Normal University, and Sun Yat-sen University.

\section{Measures}

\section{Postgraduate Mental Health Scale}

The research selects the "Postgraduate Mental Health Scale" compiled by Liu Yilan (2020), which contains three dimensions: positive emotional experience, positive psychological quality, and well social support.The response scale ranges from 1 (strongly disagree) to 5 (strongly agree). This instrument consists of 47 items. In the present sample,the total Cronbach a coefficient of the scale is 0.978 ,and the internal consistent reliability of each sub-scale is $0.936,0.961$ and 0.955 ,which showed high reliability.

\section{Langer Mindfulness Scale (LMS)}

This study uses the Chinese version of the Langer Mindfulness Scale (LMS) developed by Alan Langer and others,translated and revised by Hu Jing and others in 2014. The LMS scale consists of 13 items, including three factors of seeking new ideas, creating new ideas and focusing. The response scale ranges from 1 (strongly disagree) to 7 (strongly agree). LMS displayed high internal consistency (Cronbach's = 0.792).The score for each item is summed to generate the total scores of mindfulness.

\section{Life Purpose Scale}

The "Life Purpose Scale" selected for the study was compiled by Lan Gongrui and others, and was continuously revised during use. It measures the two dimensions of finding a sense of purpose in life and having a sense of purpose in life through 18 items. 5 items are reversed calculation. The response scale ranges from 1 (very inappropriate) to 7 (very appropriate),the higher score means the stronger sense of purpose in life.In the present sample,the Cronbach a coefficient of the total scale and two sub-scales are $0.811,0.563$, and 0.727 ,respectively.

\section{Procedure}

Data were collected from November 2020 to January 2021.Graduate students were asked to complete a series of questionnaires regarding mindfulness, mental heath and sense of purpose in life.They were informed that all responses would be confidential and would in no way impact their academic evaluation.

SPSS20.0 is used to analyze the data for exploratory analysis,descriptive analysis, variation test,correlation analysis and regression analysis. The process program (Hayes) is used,and the Bootstrap method is used for the significance test (Bao Zhenzhou eta,2013).

\section{Results}


Hypothesis testing is carried out on the basis of the correlation of various variables, which is based on the test method of intermediary effect proposed by Wen Zhonglin and Ye Baojuan (2014),the mediating role of the sense of purpose in life between mindfulness and the mental health of graduate students is tested,and the total effect of mindfulness on the mental health of graduate students is examined.The results are shown in the Table 1.The hypothetical intermediary model does not contain "0" in the 95\% confidence interval. Mindfulness can positively predict the sense of purpose in life; the sense of purpose in life can positively predict the mental health of graduate students;and mindfulness can also positively predict the mental health of graduate students. The sense of purpose in life has a significant mediating effect between mindfulness and the mental health of graduate students. The direct effect of specializing in the mental health of graduate students is 0.0374 , the indirect effect is $0.0268 \times 0.0561=0.0015$; the total effect is: 0.0366 ; the ratio of the indirect effect to the total effect is 0.041 , that is, the mindfulness of high school students has $4.1 \%$ influence on the mental health of those through career adaptation.

Table 1.The mediating and regulating of the model

\begin{tabular}{|c|c|c|c|c|}
\hline Variable & Estimate & Lower & Upper & $P$ \\
\hline Mindfulness $\rightarrow$ Sense of purpose in life & .0268 & .3299 & .4351 & $.000 * \star \star$ \\
\hline Sense of purpose in life $\rightarrow$ Mental health & .0561 & .6397 & .8602 & $.000 * \star \star$ \\
\hline Mindfulness $\rightarrow$ Mental health & .0374 & .1608 & .3080 & $.000 * \star \star$ \\
\hline family economic condition $\times$ Mindfulness $\rightarrow$ Mental health & .0688 & -.2956 & -.0250 & $.0204^{*}$ \\
\hline Type of degreexMindfulness $\rightarrow$ Sense of purpose in life & .0537 & -.2601 & -.0491 & $.004^{\star \star}$ \\
\hline $\begin{array}{l}\text { Professional interest } \times \text { Sense of purpose in life } \rightarrow \text { Mental } \\
\text { health }\end{array}$ & .1605 & .3624 & .9933 & $.000 * \star *$ \\
\hline
\end{tabular}

Further, the differences of demographic variables in each path of the model were tested. The results show those are significantly different in family economic status and type of degree and professional interest.The performance is that the better the family economic situation, the weaker the influence of mindfulness on their mental health. The mindfulness of academic grads has a greater impact on their sense of purpose in life than that of professional grads. In addition, the higher professional interest of graduate students has less impact on the effect of the sense of purpose in life on mental health of graduate students.

\section{Discussion}

\section{Mediating roles of the sense of purpose in life}

As is hypothesized,the present study demonstrates that mindfulness has a direct impact on the mental health of graduate students, and the sense of purpose in life has a partial mediating effect effect on 
concentrating and the mental health of graduate students.Mindfulness not only directly affects the mental health of graduate students, but also can further affect the mental health of graduate students through the sense of purpose in life.The intermediary model can be explained from the following perspectives: On the one hand,from the perspective of social cognition,mindfulness is the characteristics of individuals that maintain a high degree of participation and sensitivity to recognize,reconstruct and innovate and recognize in social situations. It is a mode of thinking formed by continuous debugging under the influence of cognition. The components of open thinking and acceptance of new things in its ego structure provide for cognition and ability for individuals in the process of seeking life goals. Individual's intentions can be extensively excavated to perceive the social environment through an open mind and thought transformations,then they can stimulate the internal motivation to realize personal values and gradually try to influence the external world in the social interaction of realizing their intentions. The whole process is added to more powerful positive factors in the formation of the purpose of life,so that it becomes more comprehensive and efficient. On the other hand, the dual-factor model of mental health indicates that happiness is an important indicator of individual mental health, and the purpose of life is the optimal key factor in the study of happiness, which shows that sense of purpose in life can strongly predict mental health.In this study, the effect size of the sense of purpose in life on the mental health of graduate students is 0.0561 , which can generally explain the phenomenon. A clear sense of purpose in life can enable graduate students to actively acquire positive factors from the social interaction environment and accumulate richer positive experiences and social support, thereby driving graduate students to achieve better self-development and maintain a high level of mental health.

\section{Regulating effect of demographic Variables}

The study found that some demographic factors have a moderating effect on the research model. The effect of mindfulness on mental health of graduate students can significantly be regulated by the family economic conditions, and the effect of mindfulness on mental health is the smallest and insignificant for graduate students from richer families. This moderating effect shows that the family economic status affects the effect of mindfulness on the mental health of graduate students. In the dual-factor model of mental health,the main factor representing negative experience is depression, and family economic status is an indicator of the survival of family functions. Studies have shown that the interaction between mindfulness and family conditions can significantly inhibit individual depression and alleviate the damage caused by insufficient family function. The interaction also promote the acquisition of positive emotional experiences (Liu Xiaofang, 2020). In the model of this study, graduate students from poor families need more active awareness and self-adjustment to obtain positive emotional experience and good social support. On the contrary, for graduate students from wealthier families,more social support can be gotten and a wider range of sources of positive emotional experiences can be obtained. Consequently, it is not obvious to promote mental health by mindfulness. In addition, the effect that mindfulness have on the sense of purpose in life can significantly be adjusted by the type of degree, due to the large gap between the academic system and training methods of academic master's degree and those of professional postgraduate.Academic graduate students focus on the deep system learning of subject theory, while professional graduate students focus on the application of system theory. The 
differences of the two types of degree compared, the cultivation of academic master's degree is more value-oriented and requires graduate students to create new things from multiple perspectives. At the same time,there are more students to become the academic postgraduate, who are recommended and extremely interested in professional subjects or have plans for their own professional development. Their sense of purpose in life is more stable than that of professional graduate students. They have a wide range of development and they judge their own purpose in life more decisively. Finally, the effect of sense of purpose in life on the mental health of graduate students can be adjusted by the interest in the chosen major. The role of graduate students' sense of purpose in life and mental health is mainly realized through happiness brought by the sense of purpose in life and the positive experience gained in the process of actively seeking self-worth and social study. Therefore, students who choose their majors based on their professional interests have more initiative and focus in their academic development, which strengthen the sense of purpose in life and continues to give graduate students motivation and incentive to achieve higher levels of achievement (Lan Gongrui, 2017).

\section{Declarations}

\section{Data Availability Statement}

The raw data supporting the conclusions of this article will be made available by the authors, without undue reservation.

\section{Ethics statement}

This study was approved by the ethics committee of Jiangsu Second normal University. The participants were informed about the purpose of the study and, prior to the start of the study, were assured that their privacy would be protected. An anonymous self-administered questionnaire was completed by students voluntarily. Written informed consent was obtained from all participants. All methods in the current study were carried out in accordance with relevant guidelines and regulations.

\section{Consent for publication}

Not applicable.

\section{Competing interests}

The authors declare that they have no competing interests.

\section{Funding}

The present study was supported by Shaanxi Province Education Science Research Institute, the project for"13th Five-Year Plan" , Approved in 2020[Grant number SGH20Q222].

\section{Contributions}


YW analyzed the data, and wrote the manuscript. TT conducted the survey and collected the data. JW conducted the statistical analysis. TT conceived the study, and reviewed the manuscript. All authors approved the final manuscript.

\section{Acknowledgements}

The authors wish to thank Demin Qian for support the survey.

\section{References}

1.Susan T. Charles, Melissa M. Karnaze \& Frances M. Leslie.Positive factors related to graduate student mental health, Journal of American College Health, 2021:1-9.

2.Özkan Çıkrıkçı, Başaran Gençdoğan. Clarifying the associations among belongingness, setting life goals, need satisfaction and positive orientations: The model testing in accordance with the attachment styles.Current Psychology. 2020, :1-16.

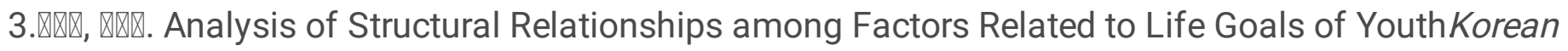
Journal of Youth Studies.2020, 27(1):191-223.

4.Meng Xuan Zhang, Xinrui Wang, Shu M. Yu, et al. Purpose in life, social support, and internet gaming disorder among Chinese university students: A 1-year follow-up study.Addictive Behaviors.2019, 99.

5.Mahama, Inuusah, Kwaw, Regine, Mensah, Kwame Jonathan, et al. Relationship between Creative Thinking and Students Academic Performance in English Language and Mathematics: The Moderating Role of Gender.Journal of Education, Society and Behavioral Science.2019:1-10.

6.Tian,Wang.The Value Orientation of Mental Health Education for graduate student under the Background of Education Modernization.Journal of Mudanjiang College of Education.2020(12):84-87.

7.Wang.Y.B,Chen.H.Y.From "problem-oriented" to "service-guided": a development for the value of mental health education.Education Review.2020(8):88-95.

8.Wang.X.Q,Zhang.Dajun.Review and Prospect of Mental Health Dual Factor Model.Special Education in China,2011(10):68-73.

9.Lan,Li,Gai.Purpose in Life: a psychological structure that indicates positive development.Progress in Psychological Science,2017,25(12):2192-2202.

10.Hu,Langer.Preliminary Revision of the LMS for Teachers in Primary and Secondary Schools. Chinese Journal of Clinical Psychology ,2014,22(01):69-73.

11.Rebecca R. Fried, Shazya Karmali \& Jennifer D. Irwin (2020) Minding many minds: An assessment of mental health and resilience among undergraduate and graduate students; a mixed methods exploratory 
study.Journal of American College Health.2020(7)

12.Yuan $\mathrm{Pu}$, Tong Xiaofang. A Special Thinking on the Influence of Talent Creativity in Mind.Journal of Ningbo University (Educational Science Edition).2019(02):1-7.

13.Zheng Aiming, Li Mei. Exploration on Mental Health Education for Graduate Students from the Perspective of Positive Psychology. Journal of Jiangsu normal University (philosophy and Social Sciences Edition).2014(06):153-156.

14.Grady RK, La Touche R, Oslawski-Lopez J, Powers A, Simacek K. Betwixt and Between: The Social Position and Stress Experiences of Graduate Students. Teaching Sociology. 2014;42(1):5-16. doi:10.1177/0092055X13502182

15.Sun Ying. on the Purpose of Life .Zhejiang Social Sciences ,2002(04):114-118.

16.Liu Yilan. Compilation and Application of Mental Health Scale for graduate student. Students Inner Mongolia normal University,2020.

17.Morgan TL, McFadden T, Fortier MS, Tomasone JR, Sweet SN. Positive mental health and burnout in first to fourth year medical students. Health Education Journal. 2020;79(8):948-962. doi:10.1177/0017896920944206

18.Eisenberg D, Downs MF, Golberstein E, Zivin K. Stigma and Help Seeking for Mental Health Among College Students. Medical Care Research and Review. 2009;66(5):522-541.

doi:10.1177/1077558709335173

19.Vorontsova-Wenger O, Ghisletta P, Ababkov V, Barisnikov K. Relationship Between Mindfulness, Psychopathological Symptoms, and Academic Performance in University Students. Psychological Reports. January 2020. doi:10.1177/0033294119899906

20.Cole NN, Nonterah CW, Utsey SO, et al. Predictor and Moderator Effects of Ego Resilience and Mindfulness on the Relationship Between Academic Stress and Psychological Well-Being in a Sample of Ghanaian College Students. Journal of Black Psychology. 2015;41(4):340-357.

doi:10.1177/0095798414537939

21.Lin Hua. New Progress of Self-determination Theory -- Motivational Theory. Journal of Hunan Institute of Science and Technology.2008(03):72-73.

22.Liu Wanlun, Tang quietly. A Study on the Relationship between Mindfulness Level and Subjective Wellbeing of College Students. Journal of Mudanjiang normal University (Social Sciences Edition).2019(05):105-113.

23. Ding Wenjie. . On the Relationship between Job Burnout and Job Engagement of Primary and Secondary School Teachers .Qingdao University ,2019. 
24.Yang Wenjing. A Study on the Research of Academic graduate student . Henan University ,2019.

25.Thoits PA. Role-Identity Salience, Purpose and Meaning in Life, and Well-Being among Volunteers. Social Psychology Quarterly. 2012;75(4):360-384. doi:10.1177/0190272512459662

26.Ulmer A, Range LM, Smith PC. Purpose in Life: A Moderator of Recovery from Bereavement. OMEGA Journal of Death and Dying. 1991;23(4):279-289. doi:10.2190/33VU-BANW-C54V-W7VF

27. Hooker SA, Masters KS, Park CL. A Meaningful Life is a Healthy Life: A Conceptual Model Linking Meaning and Meaning Salience to Health. Review of General Psychology. 2018;22(1):11-24. doi:10.1037/gpr0000115

28.McKnight PE, Kashdan TB. Purpose in Life as a System that Creates and Sustains Health and WellBeing: An Integrative, Testable Theory. Review of General Psychology. 2009;13(3):242-251.

doi:10.1037/a0017152

29.Small JL. Understanding Change: Spirituality, Faith, Religion, and Life Purpose in Student Affairs. About Campus. 2014;19(2):11-16. doi:10.1002/abc.21151

30.Hanna Suh, Shin Ye Kim \& Eleanor A. McCabe (2020) Profiles of mindfulness and difficulties in emotion regulation and links to work-family-school conflict, Journal of American College Health, DOI: $10.1080 / 07448481.2020 .1752696$

\section{Figures}

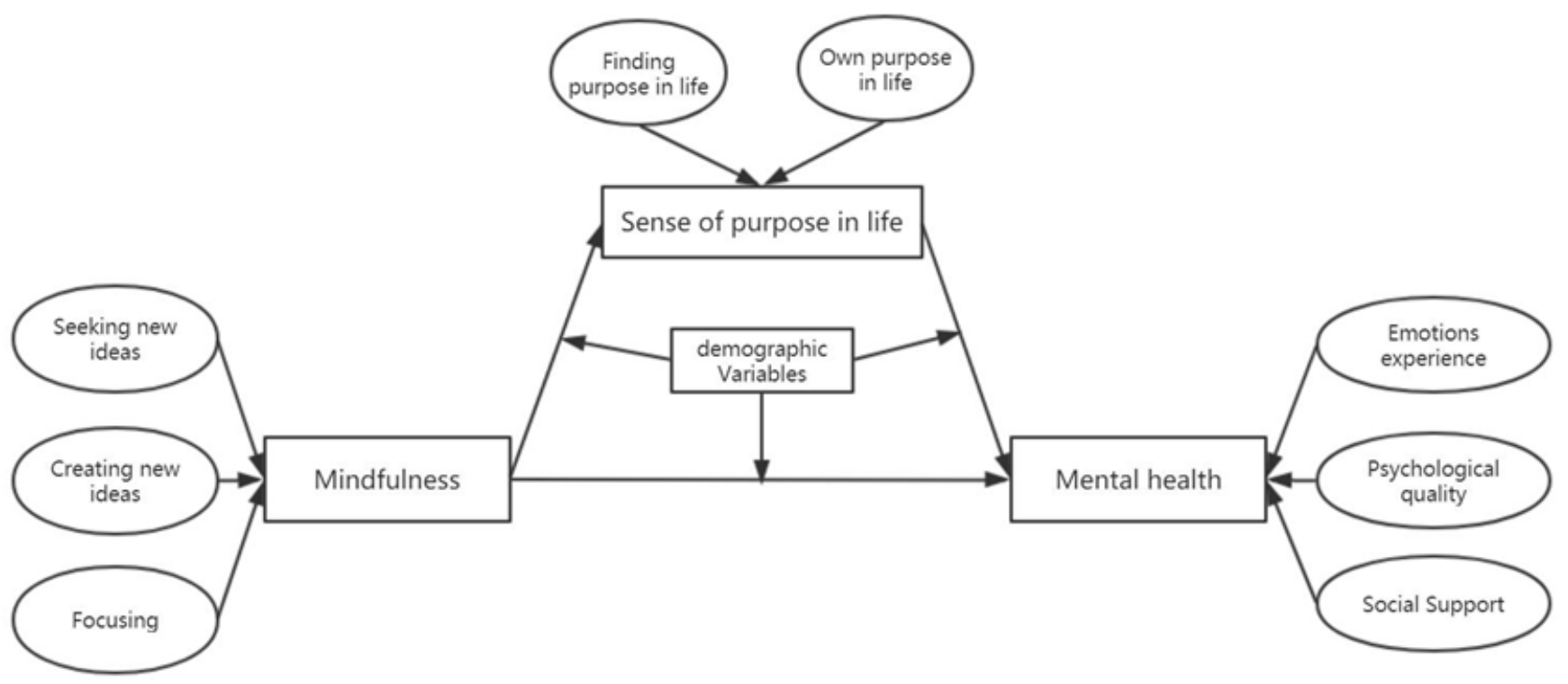

\section{Figure 1}

The model of this study 